\title{
A MODEL OF ASSORTATIVE MATING WITH PARTIAL DOMINANCE
}

\author{
A. E. STARK \\ School of Community Medicine, The University of New South Wales, P.O. Box 1, Kensington, \\ N.S.W., Australia, 2033
}

Received 14.x.76

\section{SUMMARY}

\begin{abstract}
A model of assortative mating incorporating partial dominance is proposed for a single locus with two alleles. It is derived by starting from an arbitrary genotypic distribution and finding symmetric and non-selective mating frequencies which duplicate this distribution. Numerical values are imputed to genotypes, the homozygotes having numerically equal values, opposite in sign, and the heterozygote having a value determined by the gene and heterozygote frequencies. The model is specified in a canonical form which reveals the correlation between mates based on genotypic values, and relates the correlation to the fixation index. It permits negative as well as positive values of the fixation index. It is shown that this general model includes several particular cases, in equilibrium phase, occurring in the literature.
\end{abstract}

\section{INTRODUGTION}

ONE of the standard models of assortative mating is that put forward by O'Donald (1960) in which one gene is dominant and a fraction of unions take place between like phenotypes and the remainder take place at random.

The model given here has some features which may make it a useful alternative; first, it includes partial dominance and, second, it allows a degree of negative assortative mating. In addition it embraces models with particular qualitative properties, for example one in which matings between like homozygotes are excluded.

It has been developed by exploiting the use of a canonical form to define mating frequencies, along the lines of that used by Stark (1976). The canonical form reveals the relation between the correlation of mates and the fixation index. As will be seen, the model is derived by postulating a genotypic distribution and then finding the representation which duplicates that distribution, in contrast to the usual method of postulating a mating system and then studying its approach to equilibrium.

\section{Definition of the mating system}

The genes at the locus in question are denoted $g$ and $G$ and the genotypes $g g, g G$ and $G G$ are assumed to have values $-a, d$ and $a$ respectively. The frequencies of genes are denoted $q$ and $p$ respectively and of genotypes are denoted $f_{0}, f_{1}$ and $f_{2}$ respectively, the same for each sex. The genotypic frequencies can be expressed in the form

$$
f_{0}=q^{2}+F p q, \quad f_{1}=2 p q-2 F p q, \quad \text { and } \quad f_{2}=p^{2}+F p q,
$$

which is used below. 
The genotypic values are centred by making them satisfy the requirement

$$
-a f_{0}+d f_{1}+a f_{2}=0 .
$$

Then $d$ can be expressed in terms of $a$ by the formula

$$
d=a(q-p) / f_{1},
$$

and centred genotypic values can be written as the vector

$$
\left\{-a, a(q-p) / f_{1}, a\right\} \text {. }
$$
$S$, is

The variance of centred genotypic values, with respect to $\left\{f_{i}\right\}$, denoted

$$
\begin{aligned}
S & =a^{2} f_{0}+\left[a^{2}(q-p)^{2} / f_{1}^{2}\right] f_{1}+a^{2} f_{2} \\
& =a^{2}\left[f_{0} f_{1}+(q-p)^{2}+f_{1} f_{2}\right] / f_{1} \\
& =a^{2} T / f_{1},
\end{aligned}
$$

say, where

$$
T=f_{0} f_{1}+(q-p)^{2}+f_{1} f_{2} .
$$

The vector of centred values is then standardised by $S^{\mathbf{z}}$ to form a vector $\left\{x_{i}\right\}$ whose elements are

$$
\begin{aligned}
& x_{0}=-\left(f_{1} / T\right)^{\frac{1}{2}} \\
& x_{1}=(q-p) /\left(f_{1} T\right)^{\frac{1}{2}} \\
& x_{2}=\left(f_{1} / T\right)^{\frac{1}{2}} .
\end{aligned}
$$

If the vector $\left\{x_{i}\right\}$ is considered as a row vector, then the conventional matrix product, denoted $\{x\}^{T}\{x\}$, of the transpose of $\left\{x_{i}\right\}$ and $\left\{x_{i}\right\}$ itself yields the following $3 \times 3$ matrix:

$$
\begin{aligned}
\{x\}^{T}\{x\} & =\left[\begin{array}{l}
x_{0} \\
x_{1} \\
x_{2}
\end{array}\right]\left[\begin{array}{lll}
x_{0} & x_{1} & x_{2}
\end{array}\right]=\left[\begin{array}{lll}
x_{0}^{2} & x_{0} x_{1} & x_{0} x_{2} \\
x_{1} x_{0} & x_{1}^{2} & x_{1} x_{2} \\
x_{2} x_{0} & x_{2} x_{1} & x_{2}^{2}
\end{array}\right] \\
& =\left[\begin{array}{lll}
f_{1} / T & (p-q) / T & -f_{1} / T \\
(p-q) / T & (q-p)^{2} / f_{1} T & (q-p) T \\
-f_{1} / T & (q-p) / T & f_{1} / T
\end{array}\right]
\end{aligned}
$$

This matrix is used later to derive an interval for $F$ which ensures that the frequencies of all mating pairs are non-negative.

Now suppose that the mating frequencies $f_{i j}$, where the first subscript is the index of female parents and the second that of male parents, are given by

$$
f_{i j}=f_{i} f_{j}\left(1+\rho x_{i} x_{j}\right), \quad(i=0,1,2 ; j=0,1,2) .
$$

The set of frequencies (2.3) shows that the mating system is symmetric with respect to male and female parents, and non-selective in that the distribution of genotypes among parents is the same as that among the population from 
which they are drawn. Therefore the gene frequencies among offspring are the same as those among parents.

The frequency of genotype $g g$ among offspring is equal to

$$
f_{0}^{2}\left(1+\rho x_{0}^{2}\right)+f_{0} f_{1}\left(1+\rho x_{0} x_{1}\right)+\frac{1}{4} f_{1}^{2}\left(1+\rho x_{1}^{2}\right)=q^{2}+\rho\left(f_{0} x_{0}+\frac{1}{2} f_{1} x_{1}\right)^{2} .
$$

Now for equilibrium it is necessary that the above frequency should be equal to $f_{0}$. Therefore $\rho$ should conform to the identity

$$
\begin{aligned}
\rho & =F p q /\left(f_{0} x_{0}+\frac{1}{2} f_{1} x_{1}\right)^{2} \\
& =4 F p q T /\left[f_{1}\left(1-f_{1}\right)^{2}\right] .
\end{aligned}
$$

Thus this completes the specification of system (2.3) which has the property that the genotypic distribution among offspring is the same as that of the parents.

\section{Admissible INTERVALS FOR THE PARAMETERS}

In order to ensure that genotypic frequencies $\left\{f_{i}\right\}$ and mating frequencies $\left\{f_{i j}\right\}$ are non-negative it is necessary to impose limits on $\rho$ and hence on $F$ in (2.3). The limits are symmetrical about $q=\frac{1}{2}$ so that it is sufficient to discuss the case $q \leqslant \frac{1}{2}$.

When $0<q \leqslant \frac{1}{4}$ the lower limit of $F$ is given by

$$
F=-q / p
$$

which identity gives rise to a system with the following simplified properties

$$
\begin{gathered}
f_{0}=0, \quad f_{1}=2 q, \quad f_{2}=p-q \\
T=f_{2}, \quad \rho=2 q /(q-p),
\end{gathered}
$$

and with the mating matrix

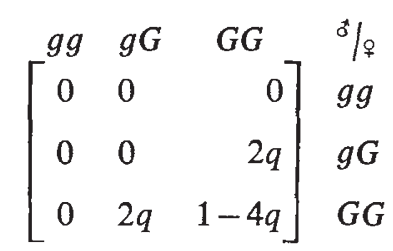

The mating matrix (3.2) is identical to that obtained under Naylor's (1962) system A, which is reached under his scheme in one round of mating. Naylor introduced system A with the statement that it "promotes the highest asymptotic heterozygosity for constant gene frequencies".

When $\frac{1}{4}<q \leqslant \frac{1}{2}$ the lower limit of $F$ corresponds to the requirement $f_{00}=0$, which, from (2.3), occurs when $1+\rho x_{0}^{2}=0$. Examination of (2.2) shows that when $f_{00}=0$ the term $f_{22}=f_{2}^{2}\left(1+\rho x_{2}^{2}\right)=0$ also, since $x_{0}^{2}=x_{2}^{2}$, in which case $F$ is given by

$$
\begin{aligned}
F & =\left[-2\left(p+q^{2}\right)+(3-4 p q)^{\frac{1}{2}}\right] /(2 p q) \\
& =\left[-2\left(q+p^{2}\right)+(3-4 p q)^{\frac{1}{2}}\right] /(2 p q) .
\end{aligned}
$$


The genotypic distribution and associated values are then:

$$
\begin{aligned}
f_{0} & =-p+\frac{1}{2}(3-4 p q)^{\frac{1}{2}} ; f_{1}=2-(3-4 p q)^{\frac{1}{2}} ; f_{2}=-q+\frac{1}{2}(3-4 p q)^{\frac{1}{2}} \\
T & =3(3-4 p q)^{\frac{1}{2}}-4 \\
\rho & =\left[17-20 p q-2(5-3 p q)(3-4 p q)^{\frac{1}{2}}\right] /\left[7-8 p q-2(2-p q)(3-4 p q)^{\frac{1}{2}}\right] .
\end{aligned}
$$

When $0<q \leqslant \frac{1}{2}$ the upper limit of $F$ corresponds to the case $f_{12}=0$, which, from (2.3), occurs under the condition

$$
f_{1}\left(1-f_{1}\right)^{2}+4 F p q(q-p)=0 .
$$

There appears to be no simple explicit solution of (3.7), but a set of numerical values is given in the table.

\section{TABLE}

Values of $\mathrm{F}$ and $\rho$ as functions of $\mathrm{q}$ when $\mathrm{f}_{12}$ and $\mathrm{f}_{01}$ are zero

\begin{tabular}{llllll}
\multicolumn{1}{c}{$q$} & \multicolumn{1}{c}{$F$} & \multicolumn{1}{c}{$\rho$} & \multicolumn{1}{c}{$q$} & \multicolumn{1}{c}{$F$} & $\rho$ \\
0 & \multicolumn{1}{c}{${ }^{3}$} & 1 & 0.3 & 0.416 & 0.863 \\
0.05 & 0.327 & 0.966 & 0.35 & 0.498 & 0.887 \\
0.1 & 0.325 & 0.933 & 0.4 & 0.628 & 0.934 \\
0.15 & 0.329 & 0.903 & 0.45 & 0.803 & 0.981 \\
0.2 & 0.342 & 0.977 & 0.5 & 1 & 1 \\
0.25 & 0.368 & 0.862 & & &
\end{tabular}

The terms $f_{02}$ and $f_{20}$ are zero if and only if $q=\frac{1}{2}$ and $F=1$, or either $f_{0}=0$ or $f_{2}=0$. This can be seen from (2.3), since the numerator of $1-\rho f_{1} \mathrm{~T}$ is $\left(1-f_{1}\right)^{2}-4 F p q$, which, considered as a quadratic function of $F$, has as its only real root $F=1$, when $q=\frac{1}{2}$. In order that (2.3) covers this case it is necessary to define $f_{1} / \mathrm{T}=1,\left(q=\frac{1}{2}, F=1\right)$.

\section{Spegial gases of the model} follows:

When $q=\frac{1}{2}$, the elements in model (2.3) simplify considerably, as

$$
\begin{aligned}
f_{0} & =f_{2}=(1+F) / 4, \quad f_{1}=(1-F) / 2, \\
S & =a^{2}\left(1-F^{2}\right) / 4, \\
x_{0} & =-[2 /(1+F)]^{\frac{1}{2}}, \quad x_{1}=0, \quad x_{2}=[2 /(1+F)]^{\frac{1}{2}} \\
\rho & =2 F /(1+F) .
\end{aligned}
$$

It can be seen that this special case of model (2.3) reduces to the model given by Stark (1976), for the case $q=\frac{1}{2}$.

Two other special cases, which form a complementary pair, arise from the respective requirements $d=-a$ and $d=a$. Each of these defines one of the genes to be dominant. By virtue of $(2.1)$ the former $(d=-a)$ is true under the condition

giving

$$
p-q=f_{1}, \quad\left(\frac{1}{4} \leqq q \leqq \frac{1}{2}\right)
$$

and

$$
\rho=\left(1-2 p^{2}\right) /\left(2 q^{2}\right)
$$

$$
F=(2 p q-p+q) /(2 p q)
$$


In this case $f_{2}=\frac{1}{2}$, so that $f_{0}=\frac{1}{2}-p+q$. The vector $\{x\}$ is given by $\{x\}=$ $\{-1,-1,1\}$. This model is admissible if and only if $q$ is restricted to the interval $\left[\frac{1}{4}, \frac{1}{2}\right]$.

The latter $(d=a)$ is true under the condition

$$
q-p=f_{1}, \quad\left(\frac{1}{2} \leqq q \leqq \frac{3}{4}\right)
$$

giving

$$
\rho=\left(1-2 q^{2}\right) /\left(2 p^{2}\right)
$$

and

$$
F=(2 p q-q+p) /(2 p q),
$$

in which case $f_{0}=\frac{1}{2}$ and $f_{2}=\frac{1}{2}-q+p$. The vector $\{x\}$ is equal to $\{-1,1$, 1).

A particular example of the second of these two cases is that for $q=\frac{3}{4}$, when $f_{0}=f_{1}=\frac{1}{2}, f_{2}=0, \rho=-1, F=-\frac{1}{3}$, and the mating matrix is given by

$$
\left\{f_{i j}\right\}=\left[\begin{array}{ccc}
0 & \frac{1}{2} & 0 \\
\frac{1}{2} & 0 & 0 \\
0 & 0 & 0
\end{array}\right]
$$

This case is the equilibrium phase of the system of negative mating given by Moree (1953), in which one gene is dominant and the only matings which occur initially are $G G \times g g$ and $G g \times g g$. Then the next generation consists only of genotypes $G g$ and $g g$, and the next mating is all of type $g g \times g G$.

\section{References}

MOREE, R. 1953. An effect of negative assortative mating on gene frequency. Science, 118, 600-601.

NAYLOR, A. F. 1962. Mating systems which could increase heterozygosity for a pair of alleles. Am. Naturalist, 96, 51-60.

o'DONALD, P. 1960. Assortive mating in a population in which two alleles are segregating. Heredity, 15, 389-396.

stark, A. E. 1976. A model of assortative mating. Ann. Hum. Genet., 39, 455-464. 